P84 CHILD EXPERIMENTATION WITH, AND EXPOSURE TO, TOBACCO AND E-CIGARETTES: A MIXED METHODS STUDY OF PRIMARY SCHOOL CHILDREN IN WALES FROM 2007 TO 2019

1,2B Hallingberg*, 'L Angel, ${ }^{1} \mathrm{R}$ Brown, ${ }^{1} \mathrm{~L}$ Copeland, ${ }^{3} \mathrm{~L}$ Gray, ${ }^{1,4} \mathrm{G}$ Moore. ${ }^{1}$ Decipher, School of Social Sciences, Cardiff University, Cardiff, UK; ${ }^{2}$ Cardiff School of Sport and Health Sciences, Cardiff Metropolitan University, Cardiff, UK; ${ }^{3}$ MRC/CSO Social and Public Health Sciences Unit, University of Glasgow, Glasgow, UK; ${ }^{4}$ SPECTRUM Consortium, UK

\subsection{6/jech-2020-SSMabstracts. 176}

Background Children in the United Kingdom (UK) today have grown up amidst strong smoking restrictions, decreasing tobacco use and increasing e-cigarette use. It is debated whether e-cigarettes interrupt downward trends in children's smoking uptake or offer a means for adults to limit tobacco use in front of their children. This study examines change over time in experimentation with, and exposure to, smoking (2007-2019) and e-cigarettes (2014-2019).

Methods Mixed methods design combining data from four national surveys of Year 6 primary school pupils in Wales from 2007-2019 $(n=6,741)$ and qualitative data from 22 focus groups within four primary schools.

Results Parental smoking $(\mathrm{OR}=0.8595 \% \mathrm{CI}=0.78-0.94)$, child tobacco experimentation $(\mathrm{OR}=0.50,95 \% \mathrm{CI}=0.40-0.63)$, and exposure to tobacco in all locations declined over time. Exposure to e-cigarettes increased in all locations from 2014-2019, as did pupil awareness $(\mathrm{OR}=2.56,95 \% \mathrm{CI}=2.13-3.09)$, and parental use $(\mathrm{OR}=1.2695 \% \mathrm{CI}=1.01-1.57])$. Child ever e-cigarette use was unchanged $(\mathrm{OR}=0.80,95 \% \mathrm{CI}=0.58-1.12)$. Since 2014, pupils' reports that parents used only e-cigarettes increased. Qualitative research identified strong disapproval for smoking and vaping indoors among children. Pupils from smoking families were more knowledgeable about e-cigarettes, with family smoking and e-cigarette use important in shaping children's exposure to, and perceptions of, smoking and vaping.

Conclusion Children's experimentation with, and exposure to, tobacco continues to decline. Further research is needed to understand whether use of e-cigarettes in cars and homes is displacing prior smoking in these locations or being introduced into environments where smoking had already been eliminated.

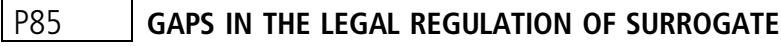 ALCOHOLS CONSUMED FOR DRINKING IN RUSSIA}

AU Gil*. Institute for Leadership and Health Management, I.M. Sechenov First Moscow State Medical University (Sechenov University), Moscow, Russia

\subsection{6/jech-2020-SSMabstracts. 177}

Background During the past fifteen years significant progress has been made in Russia in reducing the total per capita alcohol consumption, which has contributed to a reduction in total and alcohol-attributable mortality. Part of this success was due to the implementation of the control policies targeting surrogate alcohols(SAs). However, the suppression of consumption of these alcohols was uneven, and at some periods after 2005 progressed rather slowly. Until now, a number of types of SAs still remain available, affordable, and consumed for drinking by subjects with alcohol use disorders (e.g. antiseptics, not denatured eau-de-colognes, medicinal spirits). This study was purported at assessing the quality and identifying gaps in laws and regulations aimed at controlling the consumption of surrogate alcohols that were effective, newly enacted or amended in Russia between 2005 and 2019.

Methods Laws and regulations directed at SAs were identified by computer search in the Russian electronic reference systems for legislation (e.g. Consultant Plus; Garant), and on the web-sites of relevant state agencies. The content analysis of texts of regulations, evaluation of diversity of provided control policies, and of timing of their implementation, was performed.

Results Seventeen key regulatory documents (e.g. laws, decrees, orders, codes) were identified and analysed. The major identified gaps, related to control of SAs, included: 1) limited range of implemented control policies (e.g. no established minimal prices, or separate excise taxes on different types of SAs), 2)discrepancies and lack of harmonisation between different decrees and orders (e.g. while one decree banned production of medicinal tinctures in larger bottles (>25 ml of volume), another order allowed it in bottles up to $100 \mathrm{ml}$ of volume), 3)poor enforcement (e.g. existence of sizable market of counterfeit methanol-based antifreeze and windshield washer liquids despite legal ban on their production and sale), 4)recommendatory character of some regulations (e.g. denaturing is recommended,but not obligatory), 5) delayed implementation of effective policies (e.g. ban on sale of specific types of SAs(cheap spirituous lotions, tonics, bath additives, food flavour enhancers)was implemented only after the large scale outbreak of poisonings with spirituous bath additive in Irkutsk in 2016), 6)temporality of implemented policies (e.g. bans on sale of cheap SAs are effective only for6-9 months and may or may not be extended for the next periods of time), 7)overall lack of harmonization and coordination between different state agencies responsible for control of SAs (e.g. while one agency effectively suppressed consumption of specific perfumery and cosmetic alcohols, another agency responsible for medicinal spirits doesn't do this), and other gaps.

Conclusion Despite the progress achieved in controlling the consumption of specific types of surrogate alcohols, Russia still needs to do substantial work to improve quality of regulation of other types of surrogate alcohols. The fragmentary delayed control, and the reduced quality and insufficient enforcement of regulations can be partially explained by the lobby of manufacturers of surrogate alcohols, some of which are large-scale businesses capable of influencing the alcohol policy-making process.

\section{P86 MAPPING INTERVENTIONS TO REDUCE THE PUBLIC HEALTH BURDEN OF GAMBLING RELATED HARMS}

L Blank, S Baxter, H Buckley Woods, E Goyder*. School of health and Related Research, The University of Sheffield, Sheffield, UK

\subsection{6/jech-2020-SSMabstracts. 178}

Background Gambling is a highly profitable commercial activity with providers that include international corporations and governments. However, growing international recognition that gambling is a source of serious harm, and of inequity in the distribution of this harm, has led to demands for action and the production of policy documents proposing public health strategies to reduce harms at international, national and local 
levels. We aimed to identify review level evidence on interventions to address or prevent gambling related harm, to document the scope and nature of the evidence base, any gaps in the evidence, and identify policy implications, including where further development and evaluation of interventions was most urgently needed to support the development of evidenceinformed policy.

Methods Systematic mapping review and narrative synthesis of review level evidence for any intervention that aimed to address or prevent gambling related harm. Inclusion criteria included all forms of gambling; all populations (whole population; identified/self-defined gamblers and specific populations at risk (e.g. children and young people); all reported outcome measures.

Results After duplication, the searches generated 1080 records. Of 43 potential papers, 13 were excluded at the full paper stage and 30 papers were included in the review. We identified seven studies relating to whole population preventative interventions (demand reduction interventions to reduce the demand for gambling $(n=3)$, and supply reduction interventions to limit opportunities to gamble $(n=4))$. Twenty three studies provided evidence relating to targeted treatment interventions for individuals with an identified gambling addiction (therapeutic interventions $(n=12)$, pharmacological interventions $(n=4)$, self-help/ mutual support interventions $(n=5)$, and studies comparing two or more of these approaches $(n=2))$. Two further types of intervention we had expected to find were not represented in the systematic review level evidence. These were interventions to screen, identify and support individuals at risk of gambling related harm (whole population) and interventions to support ongoing recovery and prevent relapse into gambling related harm for individuals with an identified gambling addiction.

Conclusion A public health approach suggests that to reduce gambling related harm, there is potential to deliver interventions across the whole pathway - from population-level regulation of provision of opportunity to gamble to screening to identify those at risk, to targeted services for populations with an identified problem already causing harms. Whilst there is some evidence for downstream interventions, there is a dearth of evidence for wider public policy interventions suggesting that implementation of these must be accompanied by robust evaluation of effectiveness.

\section{P87 ARE PEOPLE WHO EXPERIENCE HARM FROM OTHERS' DRINKING ALCOHOL MORE LIKELY TO SMOKE? A CROSS SECTIONAL ANALYSIS OF NATIONAL SURVEY DATA}

JB Burton, MH Horton* ${ }^{*}$ CG Griffiths. Health Improvement, Public Health England, London, UK

\subsection{6/jech-2020-SSMabstracts. 179}

Background Smoking is a modifiable risk factor and has a well-known relationship with socioeconomic status and mental health. However, research into alcohol harm from others and its association with smoking habits is sparse. Despite a significant minority $(20.1 \%)$ of the population experiencing some form of harm from other's alcohol consumption there has been little study into its effects on smoking habits. In this study we aimed to compare the prevalence of smoking in those who experience harm from other's alcohol consumption and those who don't.

Methods We conducted cross-sectional analysis on the Alcohol Toolkit Study data from 2015 to 2016. Households were selected using a hybrid system of random probability sampling and simple quota sampling. Respondents aged over 16 were asked about their smoking habits and whether they had experienced harm from another's drinking in the last year. Aggressive harm was categorised to include more serious harms. We used logistic regression to explore if the characteristics of those who experienced harm including age, ethnicity, work status, disability, housing tenure, life stage, qualifications and personal drinking, account for any of the difference in smoking rates between the people who experience harm and those who don't.

Results 4881 participants were included in the analysis. 38\% of respondents that had experienced aggressive harm from others' alcohol consumption were smokers compared with $26 \%$ of those who had experienced some harm and $17 \%$ of those who had experienced no harm. Some of the variation was explained by the respondents' personal characteristics, but the odds of smoking remained higher for those who experienced harm (OR $1.2795 \mathrm{CI} \%$ 1.05-1.56) and for those who experienced aggressive harm (OR 1.92 95\%CI 1.46-2.53) compared with those who did not.

Conclusion Alcohol and tobacco use are closely linked. This exploratory analysis has allowed us to show that the link extends to people who have experienced harm from another person's drinking. People who have experienced harm are more likely to smoke, particularly for those who experience aggressive harm. The reasons for this correlation between alcohol harm and tobacco use have not been investigated before and this study shows that the relationship does not appear solely to be due to sociodemographic characteristics. This warrants further study. Smoking cessation services should take into consideration the impacts of harm from other's alcohol consumption on the smokers attempts to quit.

\section{P88 EXPLORING THE RELATIONSHIP BETWEEN SOCIAL MEDIA USE AND ALCOHOL CONSUMPTION OVER TIME AMONG YOUTHS; FINDINGS FROM UNDERSTANDING SOCIETY}

L Ng Fat* ${ }^{*}$ N Cable. Epidemiology and Public Health, University College London, London UK

\subsection{6/jech-2020-SSMabstracts. 180}

Background Given the decline in alcohol consumption amongst young people, which has coincided with the rise in technological use, there is a need for research to investigate whether technological use might be impacting on how young people drink alcohol. This study explores how social media use, and changes in social media use over time might affect alcohol consumption among youths.

Methods Using data from Understanding Standing, a UK household longitudinal study, from participants aged 12-19 $(\mathrm{N}=3586)$ from the youth and main survey in Wave 3 (2011-2013), and followed up in Wave 6 (2014-2016), we explored whether belonging to social media sites and frequency of interaction on social media sites in the past week, were related to being a drinker and drinking 